\title{
APLICATIVO MÓVEL PARA AFERIÇÃO DA FREQUÊNCIA CARDÍACA E ACOMPANHAMENTO MÉDICO REMOTAMENTE
}

\author{
A. C. Dantas*, E. B. Gouveia* e E. K. Takahashi* \\ *Universidade Federal de Uberlândia, Uberlândia, Brasil \\ e-mail: akanehar@gmail.com
}

\begin{abstract}
Resumo: Este trabalho apresenta um monitor de frequência cardíaca para dispositivos móveis que através de técnicas de processamento de imagens é possível capturar as imagens obtidas do dedo indicar do usuário e processa-las com técnicas de análise de dados removendo ruídos e assim mensurar a frequência cardíaca. As informações obtidas podem ser facilmente acessadas através do aplicativo por meio de relatórios visuais, testes realizados demonstraram resultados satisfatórios quando comparados com aparelhos que já possuem esse mecanismo implementado fisicamente.

Palavras-chave: Monitor Cardíaco, Aplicativo Médico, Aferição Cardíaca, Aplicativo móvel, Software Médico.
\end{abstract}

\begin{abstract}
This paper presents a heart rate monitor for mobile devices that can capture images obtained from the index finger through image-processing techniques and process them with data analysis techniques by removing noise and measuring the heart rate. The information obtained are easily accessed by visual reports through an application and the tests have shown satisfactory results when compared with devices that already have this mechanism physically implemented. Keywords: Cardiac Monitor, Medical application, Gauging Heart, Mobile application, Medical Software.
\end{abstract}

\section{Introdução}

Doenças cardiovasculares (DCV) são umas das principais causas de mortes em mulheres e homens no Brasil, na maioria dos casos esses incidentes estão diretamente ou indiretamente relacionados a outras doenças [1]. Tomando como base essas informações e a realidade da grande maioria da população de não realizar exames clínicos cardiovasculares com certa frequência, sendo este um grande problema da população mundial atual, desenvolvemos um aplicativo móvel capaz de fornecer aos usuários uma maneira rápida e pratica de realizar uma análise dos batimentos cardíacos e registra-los de maneira dinâmica para consultas futuras.

Os oximetros são aparelhos não invasivos bastante utilizados para medir o quanto de oxigênio seu sangue está transportando, eles podem ser encontrados atualmente em vários formatos como: pulseira, relógios, faixas e até em adesivos, a capacidade de smartphones medirem os batimentos cardíacos não é algo novo, mas para que isso seja possível é necessário na maioria das vezes a aquisição de um hardware extra, ou o aparelho já possui este recurso de fábrica sem a necessidade de aquisição pelo usuário.

Neste trabalho é proposto e desenvolvido um software capaz de transformar qualquer smartphone atual em um aparelho capaz de medir a frequência cardíaca através de técnicas de processamento de imagens nas câmeras digitais desses aparelhos. Além de possibilitar um acompanhamento remoto pelo médico do usuário ou pelo próprio sistema, uma vez que detectado anormalidades na frequência cardíaca obtida é transmitido para usuário alertas para que ele procure um médico.

Este trabalho tem como objetivo permitir o acesso de mais pessoas a este tipo de exame e tornar isso algo rotineiro e simples para todo e qualquer tipo de usuário, além de permitir uma maior interação entre médicos e pacientes de maneira simples e eficiente através do acompanhamento por um profissional. Colaborando assim de maneira significativa para a descoberta de problemas cardiovasculares na população e consequentemente a redução na taxa de mortes relacionadas a este problema.

\section{Materiais e métodos}

Para o desenvolvimento do trabalho aqui proposto foi desenvolvido um software móvel para dispositivos Android, um sistema operacional baseado em Unix especialmente projetado para dispositivos moveis além de ser o mais utilizado no mundo [3] e em mais de 190 países ao redor do mundo. Para desenvolver para este tipo de dispositivo a linguagem utilizada foi a linguagem orientada a objetos Java, atualmente ela é a linguagem oficial para desenvolvimento na plataforma. O primeiro passo para o desenvolvimento da aplicação foi a definição do fluxo da aplicação e junto a definição de como seria realizada a captura desses batimentos pela câmera do aparelho móvel.

Após a análise dos requisitos funcionais e não funcionais necessários para que a aplicação executasse de forma correta sua funcionalidade o seguinte fluxo de informação foi definido: cadastrar o usuário, realizar aferição da frequência cardíaca em batimentos do minuto (BPM) por meio de estimativa, processar essas informações que serão enviadas para o servidor de aplicação, registrar essas informações devidamente processadas, gerar relatórios visuais para o usuário de 
forma clara facilitando sua compreensão, e por último através de regras fornecer ao usuário um feedback sobre a sua saúde cardíaca, todo esse fluxo esta exemplificado na Figura 1.

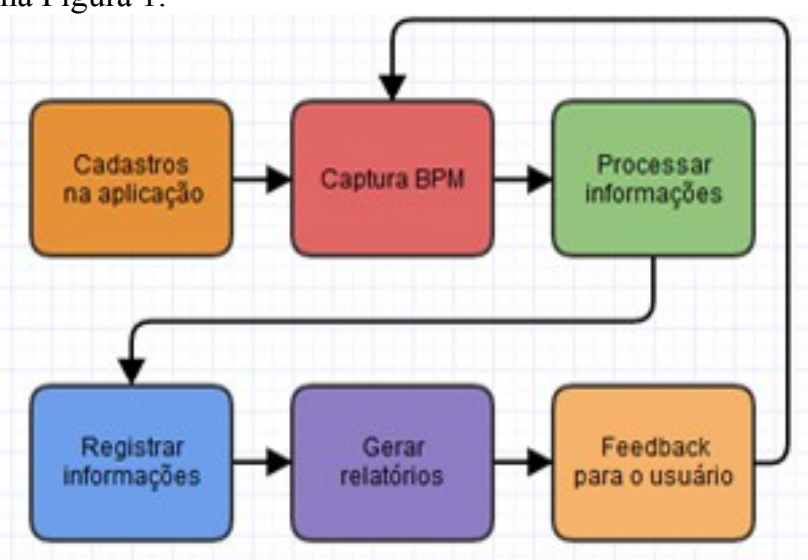

Figura 1: Fluxograma principal da aplicação.

Uma das partes mais importantes para a aplicação é a técnica utilizada para conseguir mensurar a frequência cardíaca do usuário assim como acontece nos oximetros atuais, para este trabalho decidimos utilizar a câmera do dispositivo móvel a qual o usuário deve posicionar seu dedo indicador sobre o mesmo, com o auxílio do led que é acionado no mesmo instante é possível obter a imagem com a utilização do método PreviewCallback do Android, esse método e responsável por retornar a todo momento o frame corrente da imagem. Uma vez com as imagens obtidas da sobreposição do dedo indicador sobre a câmera com o LED acionado foi necessário utilizar técnicas de processamento de imagens para se obter as variações de tons de vermelho e assim então pode mensurar a frequência cardíaca do paciente.

Aparelhos Android atualmente utilizam como padrão de cores o YUV420sp que contém respectivamente os valores de $\mathrm{Y}, \mathrm{U}, \mathrm{V}$ agrupados para compor as cores, esse modelo é exibido na Figura 2, esse padrão foi utilizado para obter a variação de tons de vermelho em cada pixel presente na imagem processada pelo aplicativo.

\begin{tabular}{|c|c|c|c|c|c|}
\hline Y1 & Y2 & Y3 & Y4 & Y5 & Y6 \\
\hline Y7 & Y8 & Y9 & Y10 & Y11 & Y12 \\
\hline Y13 & Y14 & Y15 & Y16 & Y17 & Y18 \\
\hline Y19 & Y20 & Y21 & Y22 & Y23 & Y24 \\
\hline U1 & U2 & U3 & U4 & U5 & U6 \\
\hline V1 & V2 & V3 & V4 & V5 & V6 \\
\hline
\end{tabular}

Após a obtenção desses tons através do padrão YUV420sp é realizado então a suavização exponencial básica dos dados em uma matriz de inteiros para assim descobrir o valor médio de pixels vermelhos presente na imagem. A suavização exponencial básica é bastante utilizada para a suavização de dados de series temporais funcionando como um filtro para a remoção de ruídos no processamento digital de sinais [4], a Equação 1 traz a formula padrão para a suavização exponencial simples utilizada na aplicação desenvolvida.

$$
S t=\alpha \cdot x t+(1-\alpha) . s t-1
$$

Após a definição do fluxo e a técnica para o processamento correto das imagens necessárias para a determinação da frequência cardíaca o próximo passo a ser realizado é o desenvolvimento das telas responsáveis pela interação com o usuário. A aplicação conta com as seguintes telas: tela inicial, cadastro de paciente, cadastro de médico, relatório pessoal e a tela principal para a realização da aferição da medição cardíaca, nesta tela o usuário deverá informar o número de sua identidade para que o sistema processe seus sinais biomédicos e associe ao seu perfil esse fluxo é ilustrado na Figura 3. Outra vantagem de se utilizar a numeração da identidade é que a mesma pode ser fornecida para a consulta de forma remota por um médico por exemplo.

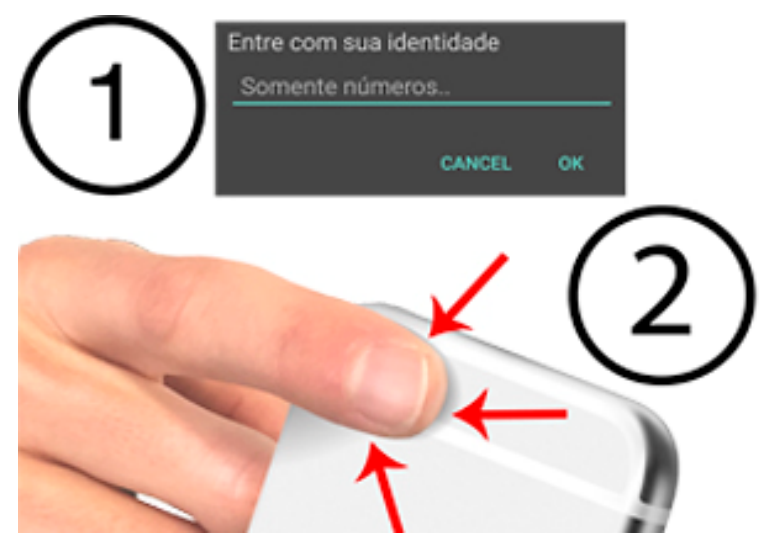

Figura 3: Passo a passo para a realização da aferição cardíaca.

Após entrar com a identidade do usuário a aplicação começa a captura das imagens para então aplicar as técnicas de processamento de imagens e suavização de dados conforme mencionado anteriormente, a Figura 4 demostra essa interface com suas periciais características.

Figura 2: Padrão de cores YUV420sp. 


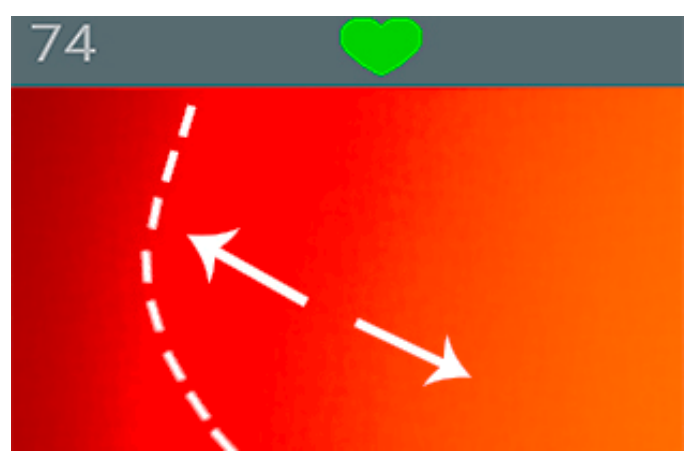

Figura 4: Interface de aferição cardíaca.

Observando a figura é possível notar a formação de duas regiões com tons de vermelho, essas regiões são formadas graças a luz emita na ponta pelo LED do aparelho móvel, essa luz alcança corrente sanguínea que está em constante fluxo sendo bombeado pelo coração, neste momento é então aplicado a suavização exponencial dos dados para se obter o valor médio de pixels vermelhos na imagem e a partir destes dados construir um vetor de frequências cardíacas.

Para obter dados mais precisos o processo é repetido por três vezes para então apresentar visualmente para o usuário a frequência cardíaca detectada naquele momento, após exibir a informação ao usuário a frequência cardíaca e a identidade informada anteriormente são enviadas para o servidor montando exclusivamente para a aplicação, a estrutura do servidor pode ser observada na Figura 5.

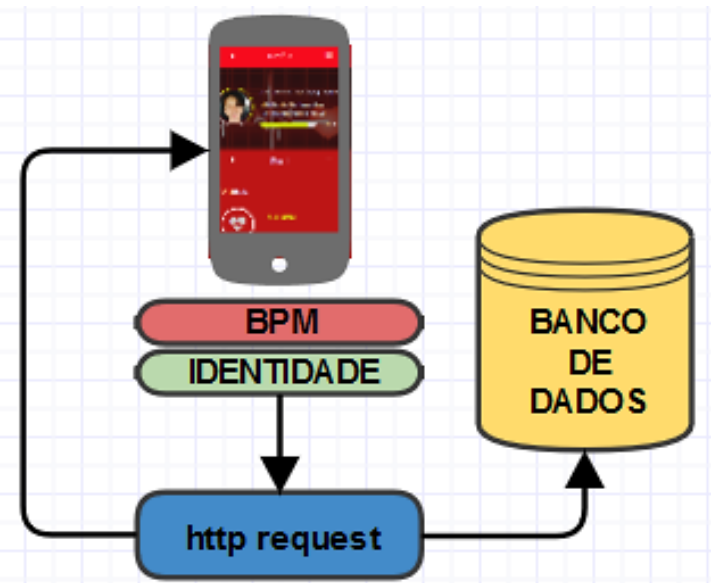

Figura 5: Arquitetura do servidor da aplicação

Para realizar o envio corretamente das informações para o servidor foi utilizado o método http request ou requisição http para trafegar os dados, chegando no servidor essas informações são armazenadas no banco de dados Mysql, um banco de dados de código aberto mais utilizado no mundo, sendo o líder, atualmente para aplicações web em geral [5]. Armazenado essas informações elas podem ser facilmente consultadas pelo paciente ou pelo médico do mesmo, além disso a aplicação realiza algumas analises informando alguma anormalidade cardíaca através de notificações no próprio aparelho.

\section{Resultados}

Com o objetivo de avaliar o desempenho da aplicação desenvolvida o mesmo foi comparado com o dispositivo físico existente no aparelho Samsung Galaxy S6, responsável por realizar a mesma funcionalidade do software aqui desenvolvido, esses testes foram importantes para demostrar a eficiência da aplicação. A variação entre as aferições foram relativamente pequenas isso ocorre porque no smartphone existe a presença de um LED infravermelho que melhora de forma satisfatória a precisão do dispositivo.

Os resultados obtidos com os testes realizados são exibidos no gráfico da Figura 6.

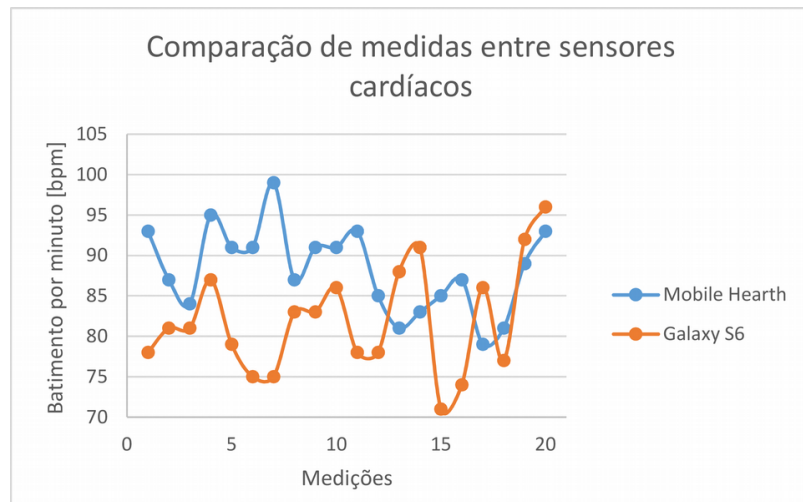

Figura 6: Comparação entre aplicativo e dispositivo.

\section{Discussão}

Os resultados obtidos foram relativamente satisfatórios e com pequenas variações, essas variações nos resultados finais são decorrentes de interferências ocasionadas por ruídos externos presentes no próprio ambiente, futuramente será testado outras técnicas de suavização e de processamento de imagens com a finalidade de melhorar ainda mais os resultados obtidos.

\section{Conclusão}

Este trabalho permitiu concluir que utilizando técnicas computacionais é possível desenvolver aplicativos móveis com funcionalidades médicas capazes de diminuir as distancias entre pacientes e médicos, através de tecnologias modernas e atuais que permitem seu acesso e acompanhamento remotamente.

\section{Referências}

[1] RIQUE, Ana Beatriz Ribeiro et al. Nutrição e exercício na prevenção e controle das doenças cardiovasculares. Rev Bras Med Esporte, v. 8, n. 6, p. 244-54, 2002.

[2] FERNANDES, Reinaldo et al. Oxímetros de pulso: operação, funcionalidade e segurança. 2001. 
[3] ANDROID. Android, the world's most popular mobile platform. 2016 . Disponível em: $<$ https://developer.android.com/about/index.html $>$.

Acesso em: 10 jul. 2016.

[4] HYNDMAN, Rob et al. Forecasting with exponential smoothing: the state space approach. Springer Science \& Business Media, 2008.

[5] MYSQL. Oracle, about. 2016. Disponível em: < https://www.mysql.com/about/>. Acesso em: 10 jul. 2016. 